\title{
Policy for Rearranging Workers for a Self-Balancing Production Line with Worker Learning
}

Daisuke Hirotani*, Katsumi Morikawa and Katsuhiko Takahashi

Faculty of Engineering, Hiroshima University, 1-4-1, Kagamiyama, Higashi-Hiroshima, 7398527, Japan

\begin{abstract}
In the traditional production lines such as assembly lines, each worker is usually assigned to a particular fixed position, and the speed of performing the task decreases until the worker masters the assigned work. However, when an imbalance in the speeds of the workers exists, any given worker can delay the overall work on the production line, and the production rate of the particular line will also decrease. To avoid this problem, the "Self-Balancing Production Line" was introduced. In this type of production line, each worker is assigned work dynamically so they can keep the production line balanced while satisfying the specific conditions. A previous paper studying worker learning has been published. When a worker learns, the speed of the worker can be increased. In that paper, the authors analyzed the conditions with and without passing and claimed that if passing is allowed, self-balancing of a production line can be achieved. However, even if the initial sequence is slowest to fastest (this is the best sequence for self-balance in the previous paper) and if passing is allowed, much more time is required to balance some conditions of speed and degree of learning (we call this the learning rate). Therefore, a new policy for rearranging workers that changes the sequence before passing should be considered for rapid balance of the production line. In this paper, the policy for rearranging workers that changes the sequence being learned is proposed, and to verify the policy, numerical experiments are performed under various conditions of speed and learning rate.
\end{abstract}

\section{Keywords: Production; Self-balancing; Learning}

\section{Introduction}

In a traditional assembly line, each worker is typically given a fixed assignment, and each worker iterates the assigned work continuously under the traditional assembly line balancing system. For this type of line, assigning workers to balance the work has been studied previously [1]. When a speed imbalance exists in this type of line, the slowest worker will delay the overall work, and the production rate of the production line will decrease. To solve this problem, a "Self-Balancing Production Line" was introduced [2]. This type of line is referred as "Bucket Brigades" and was used in at least two commercial environments: apparel manufacturing and distribution warehousing [3]. In this type of production line, each worker is assigned to work according to the worker's own pace, and when the last worker completes a task, he/she walks back and takes over the next task from his/her predecessor. The predecessor then walks back and takes over the next task from his/her predecessor; this cycle continues until the first worker walks back and starts a new task. Because faster workers are assigned more work in processing a task and vice versa, balance can be maintained. For this line with a constant working speed, the maximum production rate can be achieved if workers are sequenced from slowest to fastest [2]. Furthermore, the balance conditions with two and three workers have been obtained numerically with simulations [3], and the production line performance with $n$ workers has been analyzed mathematically [4]. There is typically assumed to be no walk-back time or hand off time in this type of line. However, Bartholdi and Eisenstein [5] consider a constant walk-back time and hand off time, and Bartholdi et al. [6] and Bratcu and Dolgui [7] consider finite walk-back speeds. Additionally, Armbruster et al. [8] consider the case in which there are two zones according to the speed (i.e., the worker's speed changes according to the zone). Under these conditions, these authors analyzed the cases in which passing is and is not allowed.

Recently, research for a self-balancing production line focused on the characteristics of the line. Lim [9] considered a U-shaped production line. In this line, the entrance and exit for an item were near each other. Therefore, the conditions for self-balance were changed. Lim analyzed the conditions for this type of line. Webster et al. [10] considered the line for order picking with respect to variations in work skills, SKU (Stock Keeping Unit) volume, and walking to the selected work content according to a pick list. This paper analyzes the impact on throughput for the above-mentioned variations.

With a few exceptions, the aforementioned studies focused on constant speed. Each worker processed at a constant speed according to the time. One factor that changes a worker's speed is learning, a topic on which many papers have been written. Relative to this paper, Nembhard [11] considers individual learning and forgetting. Under these conditions, he proposed a heuristic approach for assigning workers, and using ANOVA (ANalysis Of VAriance), he analyzed the effect of this approach. Jaber et al. [12] considered several learning and forgetting models. Using these models, these authors analyzed the effects of learning and forgetting. Shafer et al. [13] analyzed the effect of learning and forgetting in an assembly line by simulation. Villalobos et al. [14] proposed dynamic work sharing methods in assembly lines assuming certain learning curves.

The above-mentioned papers are considered without the aspect of learning. Several previous papers studying learning have been published. Armbruster et al. [15] considered learning with and without allowing one worker to pass the other. These authors claimed that if

*Corresponding author: Daisuke Hirotani, Faculty of Engineering, Hiroshima University, 1-4-1, Kagamiyama, Higashi-Hiroshima, 7398527, Japan, Tel: +81-82424-7703; Fax: +81-82-422-7024; E-mail: dhiro@hiroshima-u.ac.jp

Received August 22, 2013; Accepted September 25, 2013; Published December 15,2014

Citation: Hirotani D, Morikawa K, Takahashi K (2014) Policy for Rearranging Workers for a Self-Balancing Production Line with Worker Learning. Ind Eng Manage 3: 146. doi: 10.4172/2169-0316.1000146

Copyright: (C) 2014 Hirotani D, et al. This is an open-access article distributed under the terms of the Creative Commons Attribution License, which permits unrestricted use, distribution, and reproduction in any medium, provided the original author and source are credited. 
passing is allowed, self-balancing can be achieved. Hirotani et al. [16] considered individual learning (i.e., different degrees of learning). These authors claimed that the slowest to fastest sequence is not the best for achieving a higher production rate. However, from previous papers, even if the initial sequence is arbitrary and passing is allowed, more much time is required to balance production for some conditions of speed and degree of learning (we call this the learning rate). Therefore, a new work policy that changes the sequence before passing should be considered to balance production rapidly. In this paper, a work policy that changes the sequence with learning is proposed, and to verify the policy, numerical experiments are performed under various conditions of speed and learning rate.

This paper is organized as follows: In section 2, assumptions, and characteristics of this self-balancing production line with learning are explained. In section 3, we propose and explain work policies for learning. In section 4 , we compare the proposed policy and analyze the characteristics of the policies under various speeds and learning rates. Finally, concluding remarks are made in section 5.

\section{The Production Line}

\section{Assumptions}

In this paper, a production line with the following assumptions is considered.

- Each worker processes only one identical item sequentially.

- Workers are sequenced from one to $n$ on the production line (Figure 1), and each worker can pass over the upstream and downstream workers. This assumption is different from previous papers $[2-10,15,16]$.

- Worker $i$ processes with increasing working speed $v_{i}^{(t)}>0$ at iteration $t$. The lower and upper limit of working speed for a worker $i$ is defined as $v_{i}^{L}$ and $v_{i}^{H}$, respectively. Degree of learning (we call this learning rate in the following sections) is defined as $\tau_{i}$.

- When the last worker finishes processing an item, worker $n$ walks back to worker $n-1$ and takes over the next item from worker $n-1$. Then, worker $n-1$ walks back to worker $n-2$ and takes over the next item from worker $n-2$. Similarly, all workers walk back to their preceding worker and take over the next item from the preceding worker, and worker 1 introduces a new item into the system. The time required to walk back and take over is ignored.

- The position of worker $i$ when he/she starts to process is given by $x_{i}$ (Figure 1). Then, the position at iteration $t$ is defined as $x_{i}^{(t)}$. Note that $x_{i}^{(t)}=0$ for any iteration $t$ because the first worker always starts to process an item.

\section{Self-balancing and convergence}

The production line has been proven to maintain balance when workers are sequenced from slowest to fastest, and the working speed is constant for all workers [2]. Subsequently, the position of the workers will converge to a unique fixed point. Under these conditions, the production rate can be calculated as the sum of each worker's speed for each worker. The condition for convergence under which the line can balance for $n$ workers has been found in a previous paper [4]. However, in this paper, the worker's speed can be increased as the worker learns, so the result of the previous paper should be modified as follows:

$$
\frac{\sum_{k=1}^{i=1}(-1)^{i+k-1} v_{k}^{(t)}}{v_{n}^{(t)}}<1
$$

The difference is only the working speed with iteration $t$. Additionally we should modify the definition of convergence. In this paper, if the maximum production rate at iteration $t$ can be achieved under $v_{i}^{(t)}$, we judged that convergence had occurred. If convergence occurs, the above-mentioned fixed point can be defined as follows:

$$
x_{i}^{(t)^{*}}=\frac{\sum_{k=1}^{i=1} v_{k}^{(t)}}{\sum_{k=1}^{n} v_{k}^{(t)}}
$$

In this paper, if the difference in the fixed points is less than $\varepsilon$, we judge that convergence has occurred. Additionally, under the conditions of convergence, if condition (1) is satisfied for all workers based on the average speeds, we judge that self-balance has occurred.

\section{Learning}

In this paper, worker learning is represented as increasing the worker's working speed. Based on the previous papers [15-17], speed is defined as follows:

$$
v_{i}^{(t)}=v_{i}^{L}+\left(v_{i}^{H}-v_{i}^{L}\right)\left(1-e^{\frac{t^{\prime}}{\tau_{i}}}\right)
$$

where $t^{\prime}$ is number of workings. In this paper, we divide the task into 1000 intervals, a difference from a previous paper [15]. Additionally, the difference from the previous paper [16] is $\tau_{i}$ where $\tau_{i}$ is the same for all workers (i.e., $\tau_{\mathrm{i}}=\tau$ ) in that paper. Figure 2 shows the learning curve varying under $v_{i}^{L}=2$ and $v_{i}^{H}=4$.

\section{Worker Rearranging Policy for Learning}

In this paper, we propose the following four policies for selfbalancing a production line with learning.

\section{Only passing (Policy 1 )}

This policy is the same as in previous papers $[15,16]$. When a worker catches up with a downstream worker, the worker can pass the other worker. As mentioned, this policy can self-balance under various speeds and learning rates. A time chart for Policy 1 is shown in Figure 3. In this figure, two lines are crossed: the first worker passes the second worker.

Applying only this policy, passing seldom occurs. Instead, the worker's starting position converges to a specific point in any sequence. This phenomenon is shown in Figure 4. This figure is an orbit of worker starting positions. In this figure, the worker starting position converges three times. Therefore, more time is required to self-balance. As a result, a new rearranging policy should be considered.

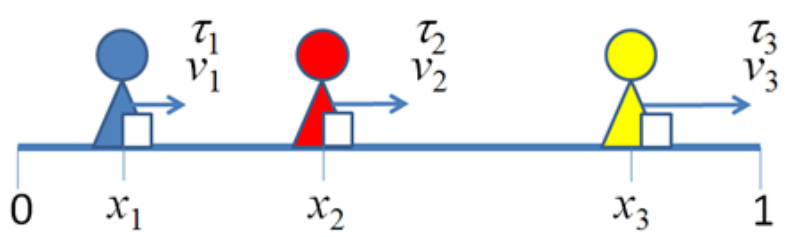

Figure 1: The production line and positions for three workers 
Citation: Hirotani D, Morikawa K, Takahashi K (2014) Policy for Rearranging Workers for a Self-Balancing Production Line with Worker Learning. Ind Eng Manage 3: 146. doi: 10.4172/2169-0316.1000146

Page 3 of 8

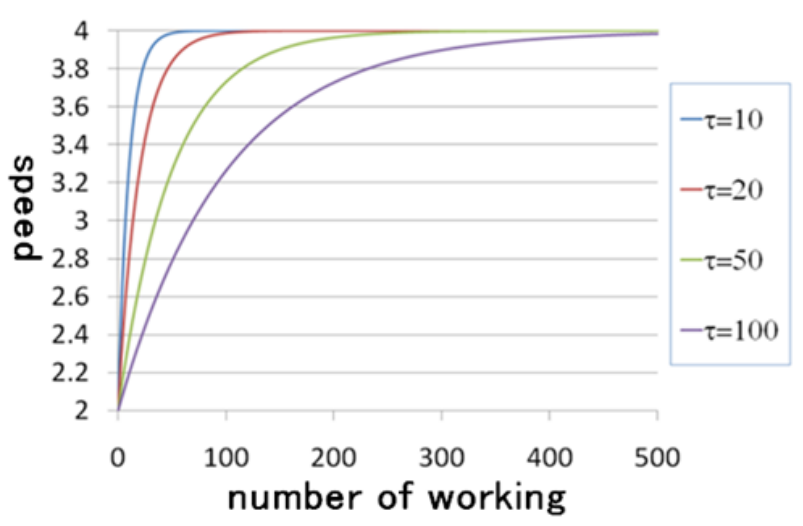

Figure 2: Learning curve with respect to number of worker under various learning rates

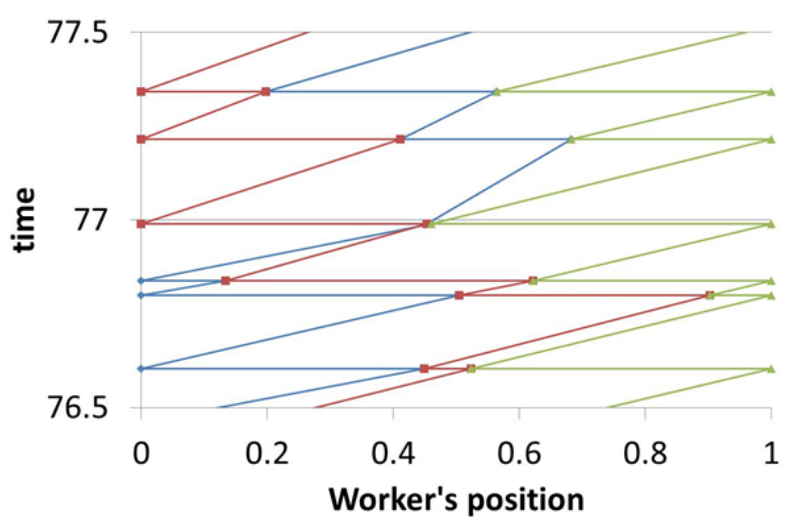

Figure 3: Time chart for Policy 1 under $_{c}=3.5, v_{2}^{H}=3, v_{3}^{H}=2.5, \tau_{1}=50$, $\tau_{2}=50, \tau_{3}=10$ and $A B C$ sequence

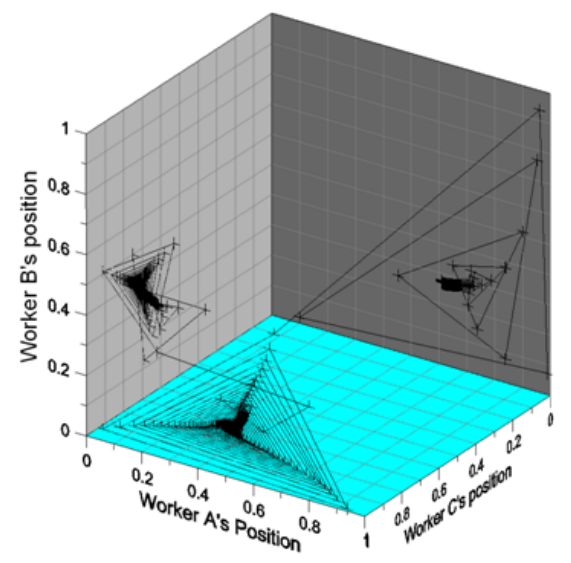

Figure 4: Orbit of worker starting positions for Policy 1 under $v_{1}^{H}=3.5$, $v_{2}^{H}=3, v_{3}^{H}=2.5, \tau_{1}=50, \tau_{2}=50, \tau_{3}=10$ and $\mathrm{ABC}$ sequence

\section{Rearranging adjacent workers (Policy 2)}

Each worker evaluates the average speed for all iterations, and if the average speed is higher than the speed of a downstream worker, the position of each worker changes to the other. This policy arises because if more time is required to pass and yet not self-balance, arranging the sequence is necessary to self-balance sooner. The time chart for
Policy 2 is shown in Figure 5. In this figure, the starting position of the first worker is changed to the starting position of the second worker. Therefore, the first worker does not take over an item.

By applying only this policy, rearranging frequently occurs because, after rearranging, speed is decreased compared to the situation before rearranging. This phenomenon is shown in Figure 6. This figure is an orbit of worker starting position. In this figure, rearranging occurs frequently for both first and second workers and second and third workers and is therefore not realistic. As a result, a new rearranging policy should be considered.

\section{Rearranging adjacent workers when workers' positions converge (Policy 3 )}

Convergence is evaluated by computing the difference between the present and the previous worker's starting positions. If these positions converge, each worker evaluates the average speed, and if the speed is higher than a downstream worker's speed, the position of each worker changes to the other's position. The difference from Policy 2 is the use of convergence. Using convergence, the worker avoids frequent changes in the position. The time chart for Policy 3 is shown in Figure 7. In this figure, the starting position of the first worker is changed to the starting position of the second worker after that worker's position converges to the specific position.

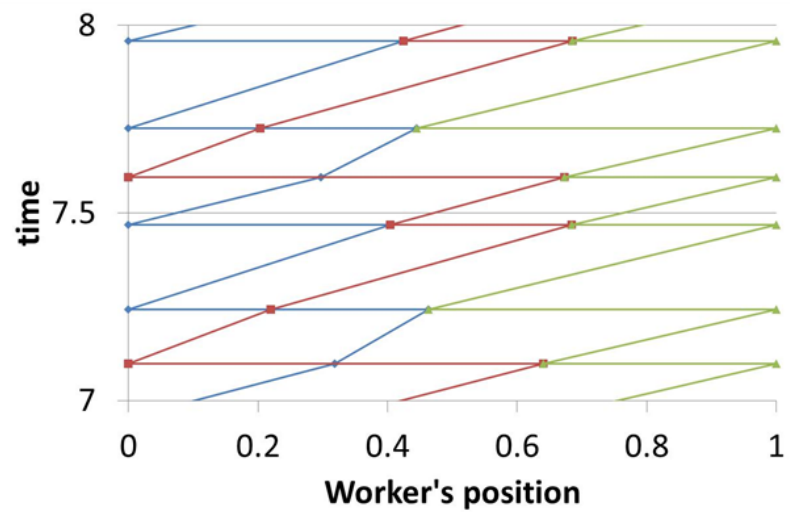

Figure 5: Time chart for Policy 2 under $v_{1}^{H}=3.5, v_{2}^{H}=3, v_{3}^{H}=2.5$, $\tau_{1}=50, \tau_{2}=50, \tau_{3}=10$ and $A B C$ sequence

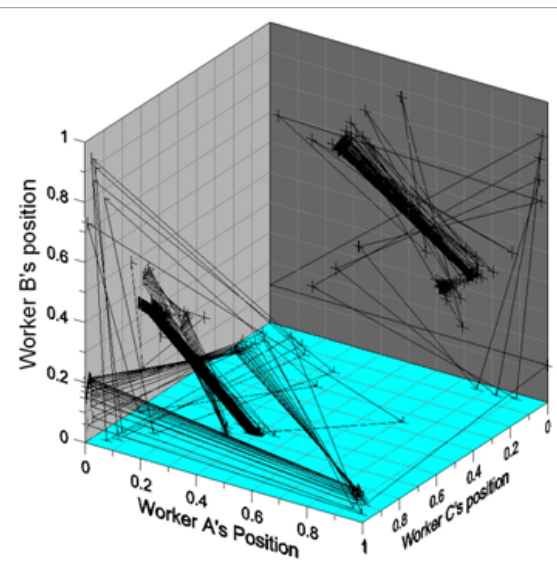

Figure 6: Orbit of worker starting positions for Policy 2 under $v_{1}^{H}=3.5$, $v_{2}^{H}=3, v_{3}^{H}=2.5, \tau_{1}=50, \tau_{2}=50, \tau_{3}=10$ and $\mathrm{ABC}$ sequence 
Citation: Hirotani D, Morikawa K, Takahashi K (2014) Policy for Rearranging Workers for a Self-Balancing Production Line with Worker Learning. Ind Eng Manage 3: 146. doi: 10.4172/2169-0316.1000146

Applying only this policy, rearrangement occurs only for adjacent workers. An orbit of starting positions for the workers is shown in Figure 8. In this figure, rearrangement seldom occurs. However, even if rearrangement occurs, the production line may have more time to self-balance because, after rearrangement, no one can be sure that the sequence after rearrangement satisfies the self-balancing condition. Therefore, as a result, a new rearrangement policy should be considered.

\section{Rearranging all workers when the worker's positions converge (Policy 4)}

Convergence is evaluated by computing the difference between the present and previous workers' starting positions. If the positions converge, each worker evaluates the average speed, and if the speed does not satisfy the self-balancing condition shown in formula (1), the starting position of each worker changes to slowest to fastest at the next iteration. The difference from Policy 3 is using the self-balancing condition. If the change to slowest to fastest is rapid, the line can selfbalance earlier. A time chart is shown in Figure 9. In this figure, after the positions converge to a specific position, the position of the first worker changes to the position of the second worker, the position of the second worker changes to the position of the third worker, and the position of third worker changes to the position of the first worker. The changed sequence is now slowest to fastest. An orbit of worker starting positions is shown in Figure 10. In this figure, rearrangement also seldom occurs.

\section{Comparing the Work Policies}

We compare four policies and evaluate these policies to determine

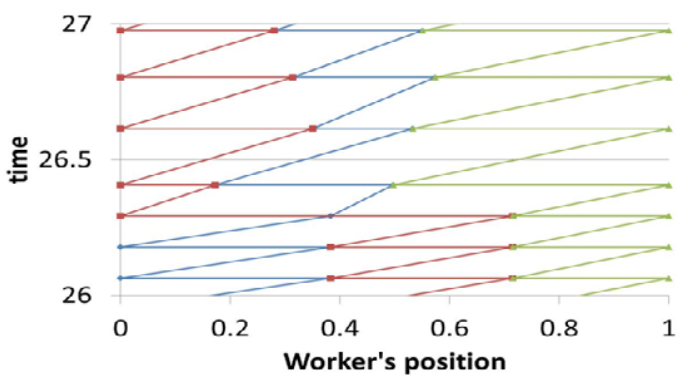

Figure 7: Time chart for Policy 3 under $v_{1}^{H}=3.5, \quad v_{2}^{H}=3, v_{3}^{H}=2.5$, $\tau_{1}=50, \tau_{2}=50, \tau_{3}=10$ and $A B C$ sequence

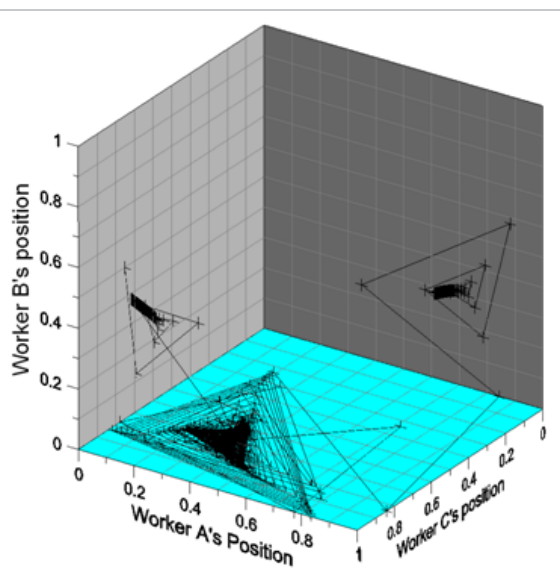

Figure 8: Orbit of worker starting positions for Policy 3 under $v_{1}^{H}=3.5$, $v_{2}^{H}=3, v_{3}^{H}=2.5, \tau_{1}=50, \tau_{2}=50, \tau_{3}=10$ and $\mathrm{ABC}$ sequence

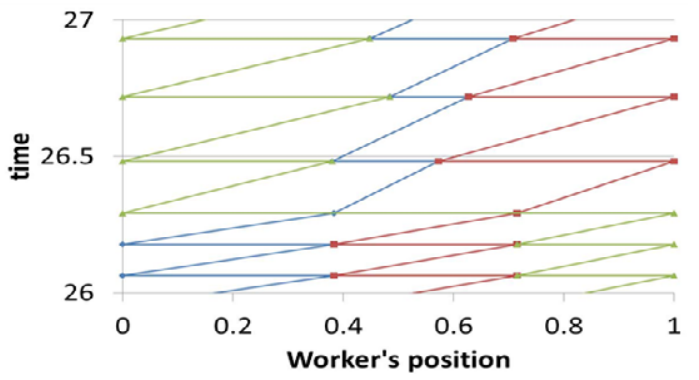

Figure 9: Time chart for Policy 4 for three workers under $v_{1}^{H}=3.5, v_{2}^{H}$ $=3, v_{3}^{H}=2.5, \tau_{1}=50, \tau_{2}=50, \tau_{3}=10$ and $\mathrm{ABC}$ sequence

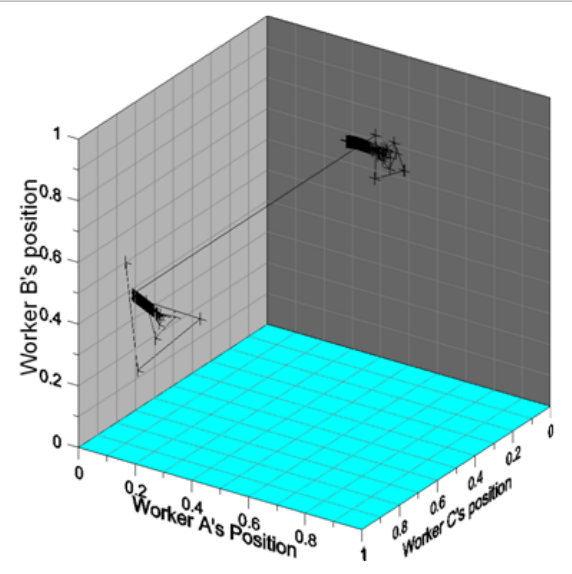

Figure 10:Orbit of worker starting positions for Policy 4 under $v_{1}^{H}$ $=3.5, v_{2}^{H}=3, v_{3}^{H}=2.5, \tau_{1}=50, \tau_{2}=50, \tau_{3}=10$ and $\mathrm{ABC}$ sequence

the best policy. We assume three workers (i.e., $n=3$ ). Therefore, the self-balancing conditions achieved by using formula (1) are $\mathrm{v}_{1}<\mathrm{v}_{3}$ and $\mathrm{v}_{1}+\mathrm{v}_{3}>\mathrm{v}_{2}$, meaning that the speed of the first worker is slower than the speed of the last worker, and the sum of the speed of the first and the last workers is faster than the speed of the second worker. Additionally, we set $\varepsilon=10^{-4}$ for evaluation of the convergence.

\section{Comparison of results}

First we evaluate the number of iterations for self-balance by varying learning rate, initial sequence and final sequence. Additionally, we evaluate the number of rearrangements (not including number of passes). For both measures, a low value is better. Because for the number of iterations, a low value means rapid convergence for selfbalancing, and for the number of rearrangements, a low value means seldom rearranging so that the worker does not have to change work range. The result for the fastest to slowest final sequence is shown in Table 1. As shown above, the fastest to slowest sequence is the worst case. Therefore, the sequence should be changed. We choose this sequence for that reason. In this table, $\mathrm{ABC}$ is a worker sequence, i.e., the first worker is $\mathrm{A}$, who has an initial speed of 1 (i.e., $v_{A}^{L}=1$ ), the second worker is $\mathrm{B}$, who has an initial speed of 1.5 (i.e., $v_{B}^{L}=1.5$ ), and the last worker is $\mathrm{C}$, who has an initial speed of 2 (i.e., $v_{C}^{L}=2$ ). In this case, the speed of the first worker (A) increases from 1 to 3.5 , the speed of the second worker increases from 1.5 to 3 , and the speed of the last worker increases from 2 to 2.5. Additionally, the term "_" indicates that this sequence does not self-balance under $\varepsilon=10^{-4}$. However, in 
analyzing this sequence, we find that there is a small difference, but the sequence seems to self-balance.

In Table 1, Policy 1 is the worst for cases of $\mathrm{ABC}, \mathrm{ACB}$, and BAC. In this case, each worker has to pass at least once. For Policy 1, the worker starting positions converge once. However, because this sequence does not satisfy the self-balancing condition later, the position frequently changes. If this behavior is repeated, the line can ultimately selfbalance, though it takes more time to do so. For the other policies, by arranging forcibly, the line can self-balance earlier.

Changing frequently occurs in Policy 2. Changing frequently is not a good situation because the worker is confused when working with frequent changing. However, the number of iterations is the best for some cases. In Policy 3, the number of iterations is not better than the number of iterations of Policy 2. On the contrary, the number of rearrangements is better than the number of rearrangements for Policy 2 because it takes more time to converge to the specific position. Additionally, to utilize the convergence state, frequent rearrangements seldom occur. In Policy 4, to overcome the disadvantages of Policies 2 and 3 , better results can be obtained because the number of rearrangements is low and the number of iterations is the best for some cases. However, for some cases, Policy 2 is the best with regard to the number of iterations. We consider why Policy 2 is the best about the number of iterations for some cases. Figure 11 shows that in one of the cases, Policy 2 is better than Policy 4. In Policy 4, rearrangement occurs several times, so it takes more time to self-balance. In this case, frequent rearrangement is better; thus, Policy 2 is better.

On the contrary, we analyze the condition where the final sequence is slowest to fastest. This sequence is the best case in a previous paper [2]. Therefore, the sequence does not have to change in some cases. This result is shown in Table 2. In Table 2, similar results can be obtained. Policy 1 is the worst for cases of BCA, CAB, and CBA. In this case, each worker has to pass at least once. For the reason as in Table 1, Policy 1 is not better. Additionally, in Policy 2, the number of rearrangements is high instead of the number of iterations being the best for some cases. In Policy 3, the number of iterations is high for cases of $\mathrm{BCA}, \mathrm{CAB}$ and $\mathrm{CBA}$, and the number of rearrangements is better than Policy 2. In Policy 4, the number of rearrangements is the best for all cases, and the number of iterations is the best for most cases. However, comparing Policies 3 and 4, the number of iterations is almost the same for cases $\mathrm{ABC}, \mathrm{ACB}$ and $\mathrm{BAC}$. We consider why this result is almost the same for the above-mentioned cases. As mentioned above, the initial sequence satisfies the self-balancing condition, as well as the final sequence. Therefore, there is no need to rearrange, and the result is almost the same for most cases of $\mathrm{ABC}, \mathrm{ACB}$ and $\mathrm{BAC}$.

Compared to the other three policies, Policy 4 is the best. At first, we compare Policy 4 with Policy 2. To avoid the use convergence, frequent rearrangement occurs. Therefore, using the convergence condition is necessary. Next, we compare Policy 3 and 4 by adding self-balancing conditions, and the number of rearrangements decreases.

As a result, Policy 4 is the best even if the number of convergence is not the best.

\section{Sensitivity analysis for the convergence condition of workers' positions}

In previous subsections, $\varepsilon=10^{-4}$. However, as seen in Figures 10 and 11 , more iteration is required for self-balance. Therefore, we perform sensitivity analysis for $\varepsilon$. $\varepsilon$ is set as $\varepsilon=10^{-2}, 10^{-3}$. The results are shown in Table 3. In Table 3, comparing to $\varepsilon=10^{-4}$, cases for BCA, CAB and
$\mathrm{CBA}$ are almost the same because there is no need to rearrange. For cases $\mathrm{ABC}, \mathrm{ACB}$, and $\mathrm{BAC}, \varepsilon=10^{-2}$ is better for most cases. We consider why $10^{-2}$ is better. Figure 12 shows orbits for worker starting position under $\varepsilon=10^{-2}$ and $\varepsilon=10^{-4}$. In this figure, for $\varepsilon=10^{-4}$, it takes more time to rearrange. Therefore, we claim that evaluation for convergence must not be set rigidly. The result is worse for some parameters because, to determine the convergence earlier, the other sequence that is not desired is changed. This circumstance is shown in Figure 13. In this figure, to change earlier, more rearrangement occurs. Therefore, roughly setting for $\varepsilon$ is not recommended. Next, as in the previous section, the case for that initial sequence of slowest to fastest is analyzed. The results are shown in Table 4. In Table 4, comparing to $\varepsilon=10^{-4}$, cases for ABC, ACB and $\mathrm{BAC}$ are almost the same because there is no need to rearrange. For cases $\mathrm{BCA}, \mathrm{CAB}$, and $\mathrm{CBA}, \varepsilon=10^{-2}$ is better for most cases for almost the same reason as previously. Therefore, we claim that evaluation for convergence must not be set rigidly. However, the result is worse for some parameters because, to determine the convergence earlier, another sequence that is not desired is changed. Therefore, roughly setting for $\varepsilon$ is not recommended.

\section{Conclusion}

In this paper, we consider the self-balancing production line with the additional factor of learning. When considering learning, more time is required for the production line to self-balance. To overcome this
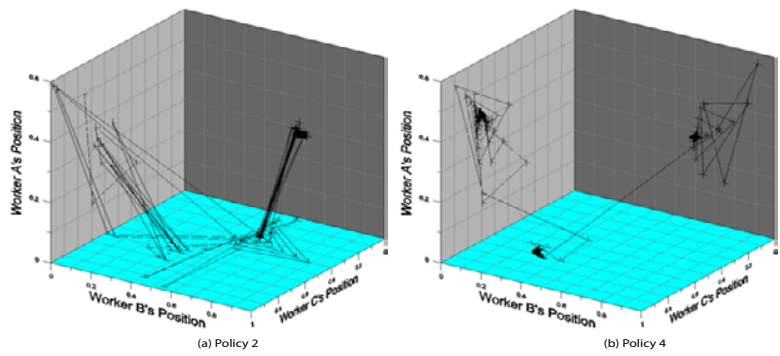

Figure 11: Orbit of worker starting positions under $v_{1}^{H}=3.5, v_{2}^{H}=3, v_{3}^{H}=2.5$, $\tau_{1}=50, \tau_{2}=50, \tau_{3}=10$ and $A B C$ sequence
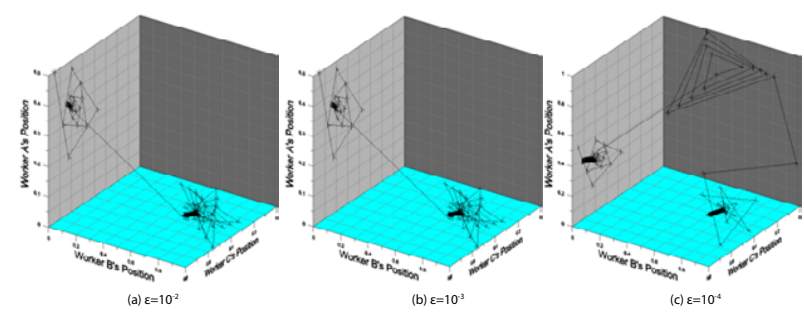

Figure 12: Orbit of worker starting positions under BAC sequence and $\tau_{1}=50, \tau_{2}=50, \tau_{3}=10$.
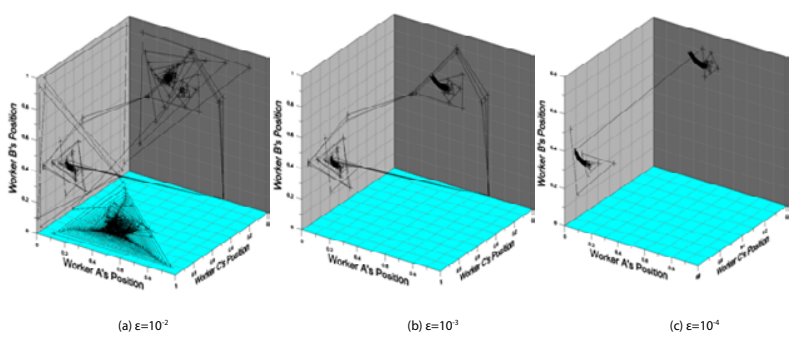

Figure 13: Orbit of worker starting positions under $A B C$ sequence and $\tau_{1}=100, \tau_{2}=50, \tau_{3}=10$ 
Citation: Hirotani D, Morikawa K, Takahashi K (2014) Policy for Rearranging Workers for a Self-Balancing Production Line with Worker Learning. Ind Eng Manage 3: 146. doi: 10.4172/2169-0316.1000146

Page 6 of 8

(a) Policy 1

\begin{tabular}{|c|c|c|c|c|c|c|c|c|c|c|c|c|c|c|}
\hline \multirow[b]{2}{*}{$\tau_{1}$} & \multirow{2}{*}{$\tau_{2}$} & \multirow{2}{*}{$\tau_{3}$} & \multicolumn{6}{|c|}{ Number of iterations } & \multicolumn{6}{|c|}{ Number of iterations } \\
\hline & & & $A B C$ & ACB & BAC & BCA & CAB & CBA & $\mathrm{ABC}$ & ACB & BAC & BCA & CAB & CBA \\
\hline 50 & 50 & 50 & 1702 & 717 & 1928 & 141 & 158 & 99 & 0 & 0 & 0 & 0 & 0 & 0 \\
\hline 10 & 50 & 50 & 1118 & 138 & 1385 & 168 & 201 & 147 & 0 & 0 & 0 & 0 & 0 & 0 \\
\hline 100 & 50 & 50 & 2478 & 1496 & 2611 & 217 & 1202 & 89 & 0 & 0 & 0 & 0 & 0 & 0 \\
\hline 50 & 10 & 50 & 1437 & 782 & 1289 & 192 & 149 & 180 & 0 & 0 & 0 & 0 & 0 & 0 \\
\hline 50 & 100 & 50 & 2505 & 564 & 3065 & 115 & 303 & 126 & 0 & 0 & 0 & 0 & 0 & 0 \\
\hline 50 & 50 & 10 & 1732 & 758 & 1980 & 327 & 1802 & 290 & 0 & 0 & 0 & 0 & 0 & 0 \\
\hline 50 & 50 & 100 & 1576 & 536 & 1786 & 233 & 175 & 237 & 0 & 0 & 0 & 0 & 0 & 0 \\
\hline
\end{tabular}

(b) Policy 2

\begin{tabular}{|c|c|c|c|c|c|c|c|c|c|c|c|c|c|c|}
\hline \multirow{2}{*}{$\tau_{1}$} & \multirow{2}{*}{$\tau_{2}$} & \multirow{2}{*}{$\tau_{3}$} & \multicolumn{6}{|c|}{ Number of iterations } & \multicolumn{6}{|c|}{ Number of rearrangements } \\
\hline & & & $A B C$ & ACB & BAC & BCA & CAB & CBA & $A B C$ & ACB & BAC & BCA & CAB & CBA \\
\hline 50 & 50 & 50 & 455 & 411 & 261 & - & 266 & 109 & 320 & 195 & 163 & 80 & 113 & 3 \\
\hline 10 & 50 & 50 & 189 & 168 & 329 & 171 & 290 & 192 & 51 & 23 & 183 & 10 & 143 & 3 \\
\hline 100 & 50 & 50 & 866 & 921 & 235 & 503 & 226 & 88 & 676 & 639 & 121 & 244 & 107 & 3 \\
\hline 50 & 10 & 50 & 458 & 443 & 350 & 331 & 130 & 174 & 341 & 291 & 176 & 142 & 26 & 95 \\
\hline 50 & 100 & 50 & 304 & 361 & 594 & 378 & 586 & 182 & 171 & 192 & 415 & 49 & 367 & 3 \\
\hline 50 & 50 & 10 & 485 & 456 & 323 & 239 & 349 & 223 & 345 & 255 & 211 & 122 & 191 & 111 \\
\hline 50 & 50 & 100 & 383 & 364 & 247 & 199 & 259 & 228 & 234 & 170 & 133 & 78 & 59 & 3 \\
\hline
\end{tabular}

(c) Policy 3

\begin{tabular}{|c|c|c|c|c|c|c|c|c|c|c|c|c|c|c|}
\hline \multirow{2}{*}{$\tau_{1}$} & \multirow{2}{*}{$\tau_{2}$} & \multirow{2}{*}{$\tau_{3}$} & \multicolumn{6}{|c|}{ Number of iterations } & \multicolumn{6}{|c|}{ Number of rearrangements } \\
\hline & & & $A B C$ & ACB & BAC & BCA & CAB & CBA & $A B C$ & ACB & BAC & BCA & CAB & CBA \\
\hline 50 & 50 & 50 & 923 & 842 & 597 & - & 332 & 179 & 6 & 7 & 5 & 1 & 1 & 1 \\
\hline 10 & 50 & 50 & 366 & 278 & 412 & 277 & 377 & 270 & 1 & 2 & 2 & 1 & 1 & 1 \\
\hline 100 & 50 & 50 & 1172 & 1582 & 693 & 464 & 377 & 435 & 6 & 15 & 2 & 1 & 2 & 2 \\
\hline 50 & 10 & 50 & 813 & 940 & 464 & 287 & 149 & - & 7 & 12 & 2 & 1 & 0 & 1 \\
\hline 50 & 100 & 50 & 1080 & 682 & 718 & 258 & 547 & 503 & 5 & 4 & 3 & 1 & 1 & 3 \\
\hline 50 & 50 & 10 & 1189 & 1075 & 545 & 691 & 575 & 290 & 2 & 8 & 3 & 4 & 2 & 0 \\
\hline 50 & 50 & 100 & 820 & 678 & 457 & 284 & 378 & 329 & 6 & 12 & 3 & 1 & 1 & 1 \\
\hline
\end{tabular}

(d) Policy 4

\begin{tabular}{|c|c|c|c|c|c|c|c|c|c|c|c|c|c|c|}
\hline \multirow{2}{*}{$\tau_{1}$} & \multirow{2}{*}{$\tau_{2}$} & \multirow{2}{*}{$\tau_{3}$} & \multicolumn{6}{|c|}{ Number of iterations } & \multicolumn{6}{|c|}{ Number of rearrangements } \\
\hline & & & $A B C$ & ACB & BAC & BCA & CAB & CBA & $A B C$ & ACB & BAC & BCA & CAB & CBA \\
\hline 50 & 50 & 50 & 302 & 308 & 299 & 141 & 158 & 99 & 1 & 1 & 1 & 0 & 0 & 0 \\
\hline 10 & 50 & 50 & 283 & 138 & 377 & 168 & 201 & 147 & 1 & 0 & 1 & 0 & 0 & 0 \\
\hline 100 & 50 & 50 & 503 & 528 & 681 & 217 & 296 & 89 & 1 & 1 & 2 & 0 & 1 & 0 \\
\hline 50 & 10 & 50 & 382 & 364 & 318 & 192 & 149 & 180 & 1 & 1 & 1 & 0 & 0 & 0 \\
\hline 50 & 100 & 50 & 429 & 276 & 530 & 115 & 303 & 126 & 1 & 1 & 1 & 0 & 0 & 0 \\
\hline 50 & 50 & 10 & 345 & 347 & 355 & 327 & 336 & 290 & 1 & 1 & 1 & 0 & 1 & 0 \\
\hline 50 & 50 & 100 & 266 & 295 & 263 & 233 & 175 & 237 & 1 & 1 & 1 & 0 & 0 & 0 \\
\hline
\end{tabular}

Table 1: Comparison with Policies under $v_{1}^{H}=3.5, v_{2}^{H}=3, v_{3}^{H}=2.5$,

(a) Policy 1

\begin{tabular}{|c|c|c|c|c|c|c|c|c|c|c|c|c|c|c|}
\hline \multirow{2}{*}{$\tau_{1}$} & \multirow{2}{*}{$\tau_{2}$} & \multirow{2}{*}{$\tau_{3}$} & \multicolumn{6}{|c|}{ Number of iterations } & \multicolumn{6}{|c|}{ Number of rearrangements } \\
\hline & & & $A B C$ & ACB & BAC & BCA & CAB & CBA & $A B C$ & ACB & BAC & BCA & CAB & CBA \\
\hline 50 & 50 & 50 & 106 & 110 & 76 & 1965 & 150 & 932 & 0 & 0 & 0 & 0 & 0 & 0 \\
\hline 10 & 50 & 50 & 290 & - & 261 & 1495 & 1699 & 761 & 0 & 0 & 0 & 0 & 0 & 0 \\
\hline 100 & 50 & 50 & 236 & 158 & 79 & 1998 & 121 & 1083 & 0 & 0 & 0 & 0 & 0 & 0 \\
\hline 50 & 10 & 50 & 145 & 158 & 116 & 2331 & 673 & 2059 & 0 & 0 & 0 & 0 & 0 & 0 \\
\hline 50 & 100 & 50 & 168 & 93 & 192 & 1128 & 3181 & 433 & 0 & 0 & 0 & 0 & 0 & 0 \\
\hline 50 & 50 & 10 & 187 & 180 & 186 & 199 & 205 & 800 & 0 & 0 & 0 & 0 & 0 & 0 \\
\hline 50 & 50 & 100 & 82 & 124 & 90 & 3864 & 149 & 2514 & 0 & 0 & 0 & 0 & 0 & 0 \\
\hline
\end{tabular}


Citation: Hirotani D, Morikawa K, Takahashi K (2014) Policy for Rearranging Workers for a Self-Balancing Production Line with Worker Learning. Ind Eng Manage 3: 146. doi: 10.4172/2169-0316.1000146

Page 7 of 8

(b) Policy 2

\begin{tabular}{|c|c|c|c|c|c|c|c|c|c|c|c|c|c|c|}
\hline \multirow[b]{2}{*}{$\tau_{1}$} & \multirow{2}{*}{$\tau_{2}$} & \multirow[b]{2}{*}{$\tau_{3}$} & \multicolumn{6}{|c|}{ Number of iterations } & \multicolumn{6}{|c|}{ Number of rearrangements } \\
\hline & & & ABC & ACB & BAC & BCA & CAB & CBA & ABC & ACB & BAC & BCA & CAB & CBA \\
\hline 50 & 50 & 50 & 106 & - & 275 & 417 & 275 & 444 & 0 & 76 & 116 & 197 & 164 & 313 \\
\hline 10 & 50 & 50 & 226 & 252 & 348 & 458 & 321 & 423 & 110 & 126 & 188 & 264 & 297 & 329 \\
\hline 100 & 50 & 50 & 236 & 202 & 260 & 370 & 258 & 393 & 00 & 76 & 60 & 167 & 131 & 229 \\
\hline 50 & 10 & 50 & 236 & 328 & 120 & 443 & 289 & 454 & 92 & 140 & 15 & 292 & 168 & 334 \\
\hline 50 & 100 & 50 & 168 & 400 & 589 & 366 & 590 & 247 & 0 & 46 & 366 & 195 & 416 & 170 \\
\hline 50 & 50 & 10 & 187 & 172 & 290 & 64 & 330 & 199 & 0 & 8 & 142 & 21 & 182 & 64 \\
\hline 50 & 50 & 100 & 82 & 506 & 230 & 921 & 238 & 861 & 0 & 242 & 108 & 633 & 124 & 673 \\
\hline
\end{tabular}

(c) Policy 3

\begin{tabular}{|c|c|c|c|c|c|c|c|c|c|c|c|c|c|c|}
\hline \multirow[b]{2}{*}{$\tau_{1}$} & \multirow[b]{2}{*}{$\tau_{2}$} & \multirow[b]{2}{*}{$\tau_{3}$} & \multicolumn{6}{|c|}{ Number of iterations } & \multicolumn{6}{|c|}{ Number of rearrangements } \\
\hline & & & ABC & ACB & BAC & BCA & CAB & CBA & $A B C$ & ACB & BAC & BCA & CAB & CBA \\
\hline 50 & 50 & 50 & 106 & 110 & 76 & 1016 & 341 & 756 & 0 & 0 & 0 & 4 & 3 & 4 \\
\hline 10 & 50 & 50 & 204 & - & 261 & 927 & 465 & 776 & 2 & 0 & 0 & 10 & 2 & 4 \\
\hline 100 & 50 & 50 & 236 & 158 & 79 & 1123 & 282 & 553 & 0 & 0 & 0 & 5 & 1 & 3 \\
\hline 50 & 10 & 50 & 145 & 158 & 116 & 933 & 471 & 882 & 0 & 0 & 0 & 10 & 4 & 4 \\
\hline 50 & 100 & 50 & 168 & 93 & 192 & 691 & 597 & 835 & 0 & 0 & 0 & 7 & 2 & 4 \\
\hline 50 & 50 & 10 & 187 & 180 & 186 & 308 & 367 & 374 & 0 & 0 & 0 & 1 & 1 & 2 \\
\hline 50 & 50 & 100 & 106 & 396 & 208 & 1726 & 282 & 1172 & 0 & 2 & 2 & 9 & 1 & 8 \\
\hline
\end{tabular}

(d) Policy 4

\begin{tabular}{|c|c|c|c|c|c|c|c|c|c|c|c|c|c|c|}
\hline \multirow[b]{2}{*}{$\tau_{1}$} & \multirow[b]{2}{*}{$\tau_{2}$} & \multirow[b]{2}{*}{$\tau_{3}$} & \multicolumn{6}{|c|}{ Number of iterations } & \multicolumn{6}{|c|}{ Number of rearrangements } \\
\hline & & & ABC & ACB & BAC & BCA & CAB & CBA & $A B C$ & ACB & BAC & BCA & CAB & CBA \\
\hline 50 & 50 & 50 & 106 & 110 & 76 & 364 & 150 & 446 & 0 & 0 & 0 & 1 & 0 & 2 \\
\hline 10 & 50 & 50 & 290 & - & 261 & 472 & 927 & 546 & 0 & 0 & 0 & 2 & 2 & 2 \\
\hline 100 & 50 & 50 & 236 & 158 & 79 & 375 & 121 & 402 & 0 & 0 & 0 & 1 & 0 & 2 \\
\hline 50 & 10 & 50 & 145 & 158 & 116 & 371 & 224 & 489 & 0 & 0 & 0 & 1 & 1 & 1 \\
\hline 50 & 100 & 50 & 168 & 93 & 192 & 300 & 448 & 600 & 0 & 0 & 0 & 1 & 3 & 4 \\
\hline 50 & 50 & 10 & 187 & 180 & 186 & 199 & 205 & 361 & 0 & 0 & 0 & 0 & 0 & 1 \\
\hline 50 & 50 & 100 & 82 & 124 & 90 & 575 & 149 & 671 & 0 & 0 & 0 & 1 & 0 & 2 \\
\hline
\end{tabular}

Table 2: Comparison with Policies under $v_{1}^{H}=2.5, \quad v_{2}^{H}=3, v_{3}^{H}=3.5$,

(a) $\varepsilon=10^{-2}$

\begin{tabular}{|c|c|c|c|c|c|c|c|c|c|c|c|c|c|c|}
\hline \multirow[b]{2}{*}{$\tau_{1}$} & \multirow{2}{*}{$\tau_{2}$} & \multirow{2}{*}{$\tau_{3}$} & \multicolumn{6}{|c|}{ Number of iterations } & \multicolumn{6}{|c|}{ Number of rearrangements } \\
\hline & & & ABC & ACB & BAC & BCA & CAB & CBA & $A B C$ & ACB & BAC & BCA & CAB & CBA \\
\hline 50 & 50 & 50 & 438 & 170 & 156 & 141 & 158 & 99 & 2 & 1 & 1 & 0 & 0 & 0 \\
\hline 10 & 50 & 50 & 190 & 138 & 263 & 168 & 201 & 147 & 2 & 0 & 1 & 0 & 0 & 0 \\
\hline 100 & 50 & 50 & 986 & 325 & 492 & 217 & 182 & 89 & 4 & 1 & 1 & 0 & 1 & 0 \\
\hline 50 & 10 & 50 & 281 & 214 & 198 & 192 & 149 & 180 & 2 & 1 & 2 & 0 & 0 & 0 \\
\hline 50 & 100 & 50 & 225 & 168 & 315 & 115 & 303 & 126 & 2 & 1 & 1 & 0 & 0 & 0 \\
\hline 50 & 50 & 10 & 447 & 243 & 100 & 327 & - & 290 & 2 & 2 & 1 & 0 & 1 & 0 \\
\hline 50 & 50 & 100 & 220 & 199 & 139 & 233 & 175 & 237 & 2 & 1 & 1 & 0 & 0 & 0 \\
\hline
\end{tabular}

(b) $\varepsilon=10^{-3}$

\begin{tabular}{|c|c|c|c|c|c|c|c|c|c|c|c|c|c|}
\hline \multirow{2}{*}{$\tau_{1}$} & $\tau_{2}$ & $\tau_{3}$ & \multicolumn{9}{|c|}{ Number of iterations } & \multicolumn{4}{c|}{ Number of rearrangements } \\
\hline 50 & 50 & 50 & 460 & 183 & 150 & 141 & 158 & 99 & 1 & 1 & 1 & 0 & 0 \\
\hline 10 & 50 & 50 & 214 & 138 & 263 & 168 & 201 & 147 & 2 & 0 & 1 & 0 & 0 \\
\hline 100 & 50 & 50 & 367 & 325 & 492 & 217 & 182 & 89 & 3 & 1 & 1 & 0 & 1 \\
\hline 50 & 10 & 50 & 381 & 232 & 206 & 192 & 149 & 180 & 2 & 1 & 2 & 0 & 0 \\
\hline 50 & 100 & 50 & 320 & 170 & 316 & 115 & 303 & 126 & 2 & 1 & 1 & 0 & 0 \\
\hline 50 & 50 & 10 & 458 & 237 & 130 & 327 & - & 290 & 2 & 2 & 1 & 0 & 1 \\
\hline 50 & 50 & 100 & 170 & 219 & 158 & 233 & 175 & 237 & 1 & 1 & 1 & 0 & 0 \\
\hline
\end{tabular}

Table 3: Sensitivity analysis for policy 4 under $v_{1}^{H}=3.5, v_{2}^{H}=3, v_{3}^{H}=2.5$, 
Citation: Hirotani D, Morikawa K, Takahashi K (2014) Policy for Rearranging Workers for a Self-Balancing Production Line with Worker Learning. Ind Eng Manage 3: 146. doi: 10.4172/2169-0316.1000146

Page 8 of 8

(a) $\varepsilon=10^{-2}$

\begin{tabular}{|c|c|c|c|c|c|c|c|c|c|c|c|c|c|c|}
\hline \multirow{2}{*}{$\tau_{1}$} & \multirow{2}{*}{$\tau_{2}$} & \multirow{2}{*}{$\tau_{3}$} & \multicolumn{6}{|c|}{ Number of iterations } & \multicolumn{6}{|c|}{ Number of rearrangements } \\
\hline & & & $A B C$ & ACB & BAC & BCA & CAB & CBA & $A B C$ & ACB & BAC & BCA & CAB & CBA \\
\hline 50 & 50 & 50 & 106 & 110 & 76 & 251 & 150 & 225 & 0 & 0 & 0 & 1 & 0 & 3 \\
\hline 10 & 50 & 50 & 290 & - & 261 & 272 & 388 & 303 & 0 & 0 & 0 & 5 & 7 & 3 \\
\hline 100 & 50 & 50 & 236 & 158 & 79 & 271 & 121 & 200 & 0 & 0 & 0 & 1 & 0 & 3 \\
\hline 50 & 10 & 50 & 145 & 158 & 116 & 291 & 203 & 335 & 0 & 0 & 0 & 1 & 1 & 1 \\
\hline 50 & 100 & 50 & 168 & 93 & 192 & 202 & 433 & 500 & 0 & 0 & 0 & 1 & 5 & 10 \\
\hline 50 & 50 & 10 & 187 & 180 & 186 & 199 & 205 & 371 & 0 & 0 & 0 & 0 & 0 & 1 \\
\hline 50 & 50 & 100 & 82 & 124 & 90 & 462 & 149 & 448 & 0 & 0 & 0 & 2 & 0 & 7 \\
\hline
\end{tabular}

(b) $\varepsilon=10^{-3}$

\begin{tabular}{|c|c|c|c|c|c|c|c|c|c|c|c|c|c|c|}
\hline \multirow{2}{*}{$\tau_{1}$} & \multirow{2}{*}{$\tau_{2}$} & \multirow{2}{*}{$\tau_{3}$} & \multicolumn{6}{|c|}{ Number of iterations } & \multicolumn{6}{|c|}{ Number of rearrangements } \\
\hline & & & $A B C$ & ACB & BAC & BCA & CAB & CBA & $A B C$ & ACB & BAC & BCA & CAB & CBA \\
\hline 50 & 50 & 50 & 106 & 110 & 76 & 251 & 150 & 247 & 0 & 0 & 0 & 1 & 0 & 2 \\
\hline 10 & 50 & 50 & 290 & - & 261 & 278 & 225 & 310 & 0 & 0 & 0 & 4 & 3 & 3 \\
\hline 100 & 50 & 50 & 236 & 158 & 79 & 271 & 121 & 221 & 0 & 0 & 0 & 1 & 0 & 2 \\
\hline 50 & 10 & 50 & 145 & 158 & 116 & 291 & 219 & 335 & 0 & 0 & 0 & 1 & 1 & 1 \\
\hline 50 & 100 & 50 & 168 & 93 & 192 & 197 & 323 & 500 & 0 & 0 & 0 & 1 & 3 & 7 \\
\hline 50 & 50 & 10 & 187 & 180 & 186 & 199 & 205 & 371 & 0 & 0 & 0 & 0 & 0 & 1 \\
\hline 50 & 50 & 100 & 82 & 124 & 90 & 464 & 149 & 489 & 0 & 0 & 0 & 2 & 0 & 5 \\
\hline
\end{tabular}

Table 4: Sensitivity analysis for Policy 4 under $v_{1}^{H}=2.5, v_{2}^{H}=3, v_{3}^{H}=3.5$,

problem, we propose four policies: only passing (Policy 1 ), rearranging adjacent workers (Policy 2), rearranging adjacent workers when workers' positions converge (Policy 3) and rearranging all workers when the workers positions' converge (Policy 4). Next, we compare the four policies according to the number of iterations of self-balancing and the number of rearrangements. As a result, Policy 4 is the best using convergence and the self-balancing conditions. Additionally, we performed sensitivity analysis of convergence condition $\varepsilon$. As a result, roughly setting for $\varepsilon$ is not recommended.

We assume only learning (i.e., worker speed is always increased). Considering the case of decreasing worker speed by forgetting, worker behavior is changed, and it is hard to self-balance compared to only increasing speed. Therefore, this topic will be studied in future work.

\section{References}

1. Scholl A (1995) Balancing and Sequencing of Assembly Lines. Physica-Verlag.

2. Bartholdi JJ, Eisenstein DD (1996) A Production Line that Balances Itself. Oper Res 44: 21-34.

3. Bartholdi JJ, Bunimovich LA (1999) Dynamics of two- and three-worker "Bucket Brigades" production lines. Oper Res 47: 488-491.

4. Hirotani D, Morikawa K, Takahashi K (2006) Analysis and Design of SelfBalancing Production Line. Computers and Industrial Engineering 50: 488-502.

5. Bartholdi JJ, Eisenstein DD (2005) Using bucket brigades to migrate from craft manufacturing to assembly lines. Manufacturing \& Service Operations Management 7: 121-129.

6. Bartholdi JJ, Eisenstein DD, Lim YF (2009) Deterministic chaos in a model of discrete manufacturing. Nav Res Log 56: 294-299.

7. Bratcu Al, Dolgui A (2009) Some new results on the analysis and simulation of bucket brigades (self-balancing production lines). Int J Prod Res 47: 369-387.

8. Armbruster D, Gel ES (2006) Bucket brigades revisited: are they always effective? Eur J Oper Res 172: 213-229.

9. Lim YF (2011) Cellular bucket brigades. Oper Res 59: 1539-1545.
10. Webster S, Ruben RA, Yang K-K (2012) Impact of storage assignment decisions on a bucket brigades order picking line. Prod Oper Manag 21: 276290.

11. Nemberd DA, (2001) Heuristic approach for assigning workers to tasks based on individual learning rates. Int J Prod Res 39: 1955-1968.

12. Jaber MY, Kher HV, Davis DJ (2003) Countering forgetting through training and deployment. Int J Prod Econ 85: 33-46.

13. Shafer SM, Nembhard DA, Uzumeri MV (2001) The effects of worker learning, forgetting, and heterogeneity on assembly line productivity. Management Science 47: 1639-1653.

14. Villalobos JR, Gutierrez MA, Mar LR, Sanchez O, Ahumada O (2011) The use of dynamic work sharing production methods to reduce the impact of labour turnover in serial assembly lines. International Journal of Manufacturing Technology and Management 23: 34-53.

15. Armbruster D, Gel ES, Murakami J (2007) Bucket Brigades with worker learning. Eur J Oper Res 176: 264-274.

16. Hirotani D, Morikawa K, Takahashi K (2009) Analysis of self-balancing production line with individual learning. Proceeding of 20th International Conference on Production Research.

17. Wright TP (1936) Factors affecting the cost of airplanes. Journal of Aeronautica Sciences 3: 122-128. 